\title{
Construção Colaborativa do Conhecimento Tecnológico, Pedagógico e do Conteúdo de Professores de Matemática ${ }^{1}$
}

\author{
Arthur B. Powell \\ Professor, Rutgers University-Newark, EUA \\ Department of Urban Education \\ powellab@andromeda.rutgers.edu
}

\begin{abstract}
Resumo
As novas tecnologias de informação e comunicação desafiam os educadores em Educação Matemática a responder às mudanças que as mesmas estimulam em nossa sociedade. Assim, baseado em dois desafios instigados pelas novas tecnologias digitais, apresentamos $^{23}$ uma proposta com o objetivo de avançar na construção colaborativa do conhecimento tecnológico, pedagógico e do conteúdo de professores de Matemática. Nossa proposta é fruto de um projeto colaborativo - $e$ Mat - entre pesquisadores de duas universidades que reúne professores de Matemática que desejam evoluir nas suas práticas, aprendendo matemática colaborativa e discursivamente com tecnologias digitais. Usamos um quadro conceitual e teórico que inclui ideias sobre o complexo de corpos de conhecimentos tecnológico, pedagógico e do conteúdo (CTPC), proposto por Mishra e Koehler (2006). Este se constitui quando um professor usa apropriadamente as tecnologias digitais para fornecer oportunidades aos seus alunos para interagirem colaborativamente para fazer Matemática (GATTEGNO, 1987). Apresentamos dados de dois momentos de um curso online que pode contribuir para o desenvolvimento profissional, ilustrando como pequenas equipes de professores constroem seu CTPC em um ambiente virtual de aprendizagem - Virtual Math Teams with GeoGebra. Assim, por meio do nosso projeto, $e$ Mat, estamos dando suporte para professores que estão dispostos a usarem novas tecnologias digitais, as quais podem ser úteis para que os seus alunos manipulem objetos matemáticos e percebam relações entre os objetos e relações de relações.
\end{abstract}

Palavras-chave: Colaboração; Conhecimento Tecnológico, Pedagógico e do Conteúdo (CTPC); Geometria Dinâmica; Aprendizagem em Ambiente Virtual;

\section{Collaborative construction of technological pedagogical content knowledge of mathematics teachers}

\footnotetext{
Abstract

${ }^{1}$ Boletim Gepem (Online) ISSN: 2176-2988 | n. 64 - Jan./Jun. 2014 (texto em diagramação).

${ }^{2}$ Este trabalho é baseado em uma pesquisa apoiada pela National Science Foundation (NSF), do programa DRK-12, sob a bolsa DRL-1118888. As conclusões e opiniões relatadas aqui são de responsabilidade do autor e não refletem necessariamente a opinião da NSF.

${ }^{3}$ Este artigo está baseado na palestra do autor no VI Congresso Internacional de Ensino da Matemática (VI CIEM, 2013). Agradecemos a Vinícius Pazuch e Edmilson Minoru Torisu, respectivamente da Ulbra e UFMG, estudantes do Programa de Doutorado Sanduíche no Exterior (PDSE) na nossa universidade, pela revisão do português.
} 
The new information and communications technologies challenge educators of mathematics education to meet the changes that it stimulates in our society. On the basis of two challenges instigated by new digital technologies, we present a response addressing the objective to evolve the collaborative construction of mathematics teachers' technological pedagogical and content knowledge. Our response is a collaborative project- $e$ Math - among researchers from two universities to engage mathematics teachers to develop practices that allow them to learn mathematics collaboratively and discursively with digital technologies. Using a conceptual and theoretical framework that includes ideas on the complex bodies of technological, pedagogical and content knowledge (Mishra and Koehler, 2006) involved when a teacher appropriately uses digital technologies to provide opportunities for students to interact collaboratively and discursively to do the mathematics (GATTEGNO, 1987). We present data from two different moments in an online professional development course to illustrate how small teams of teachers construct their CTPC in a virtual learning environment-Virtual Math Teams with GeoGebra. Through our project, $e$ Math, we are supporting teachers to be willing to adapt to new digital technologies that may be useful for your students to manipulate mathematical objects and perceive relationships between objects and relations of relations.

Keywords: Collaboration; Technological Pedagogical Content Knowledge; Dynamic Geometry; Online Learning;

\section{Novos desafios com as tecnologias digitais}

As tecnologias de informação e comunicação, como a mídia social, evoluem rapidamente e estão cada vez mais envolvidas na vida pública e privada. Essas evoluções geram novas realidades e implicam dois desafios fundamentais para a Educação Matemática. O primeiro é que os avanços das tecnologias digitais e suas inserções na sociedade requerem que pesquisadores e educadores investiguem, significativamente, os potenciais dessas novas tecnologias para a Educação. Há pesquisas que investigam o uso de fóruns e outros recursos digitais assíncronos (BAIRRAL, 2007; CHEONG e CHEUNG, 2008; LI, DONG, e HUANG, 2009; SERRADÓ, 2009, 2012). No entanto, em Educação Matemática há um número reduzido de estudos que abordam uma das potencialidades fundamentais da Web 2.0: a colaboração (BAIRRAL e POWELL, 2013; POWELL e GRISI-DICKER, 2012; POWELL e LAI, 2009). Em particular, são necessárias investigações sobre como professores de matemática de escolas dos anos finais do Ensino Fundamental e do Ensino Médio podem usar atributos da Web 2.0 para explorar problemas abertos e vivenciar esse processo numa perspectiva colaborativa ${ }^{4}$ usando aplicativos da matemática dinâmica.

O segundo desafio associado à rapidez dos avanços das tecnologias de informação e comunicação está relacionado com a necessidade de colaboração para encontrar soluções relativas ao design de projetos e desenvolvimento de novos produtos, mídias e serviços para melhorar a vida do planeta e de seus habitantes. Há três aspectos relativos à

\footnotetext{
${ }^{4}$ Há grupos de pesquisadores investigando o uso de computadores e as funcionalidades da Web 2.0 para suportar a aprendizagem colaborativa. Veja Stahl, Koschmann e Suthers (2008) para uma perspectiva histórica.
} 
produção de conhecimentos que o mundo atual privilegia: (1) a interação interpessoal e social, (2) a resolução de problemas e (3) a comunicação. No entanto, no Ensino Fundamental II e Ensino Médio, em particular, os modelos tradicionais de ensino ainda dominam o cenário de educação matemática (JACOBS et al., 2006; STIGLER e HIEBERT, 1999).

Os dois desafios comentados anteriormente estão sendo conquistados na comunidade de matemáticos. Mesmo os ganhadores da Medalha Fields pensam com outros matemáticos, publicam trabalhos junto com outros matemáticos e valorizam o trabalho colaborativo. O ganhador da Medalha Fields em 2006, Terence Chi-Shen Tao, numa discussão no blog de um ganhador da Medalha Fields em 1998, Timothy Gowers, escreveu o seguinte:

Eu não posso falar pelos outros, mas em minha própria pesquisa, pelo menos metade das minhas publicações está em conjunto com um ou mais autores, e entre as publicações que eu considero entre os meus melhores trabalhos, praticamente, são todos trabalhos conjuntos. (ALAGIE e ALAGIE, 2013, p. 28, minha tradução).

Alguns dos grandes avanços da matemática hoje em dia são resultados de colaboração. Na Educação Matemática, o trabalho colaborativo deve ser privilegiado. Considerando esses dois desafios - a tecnologia e a colaboração-para a Educação Matemática, emergem várias questões interessantes. Entre elas, elaboramos duas: Como incorporar tecnologias digitais para o ensino e a aprendizagem para ajudar os estudantes a se envolverem em suas práticas discursivas, colaborativas e matemáticas? Como professores de matemática podem envolver suas capacidades de ensinar com tecnologia para contribuir na produção de práticas discursivas, colaborativas e matemáticas de seus estudantes? Essas questões norteiam nosso projeto de investigação denominado $e$ Mat.

\section{Novas possibilidades com as tecnologias digitais}

O projeto eMat, nos Estados Unidos, é financiado pela National Science Foundation (NSF) e coordenado pelo autor. Seu propósito é contribuir com a aprendizagem de professores de matemática a desenvolverem práticas que permitam aos seus estudantes o engajamento para aprender matemática colaborativa e discursivamente com tecnologias digitais. No projeto, trabalhamos com professores do Ensino Fundamental II e Ensino Médio que posteriormente podem implementar o projeto com seus estudantes. Assim, nesta seção, apresentamos ideias teóricas que fundamentam nossa investigação, talhamos o projeto eMat, o ambiente online $V M T c G$ e discutimos dados provindos de duas atividades realizadas com os professores participantes da disciplina.

\section{Quadro conceitual e teórico}

Uma das ideias conceituais que usamos em nossa investigação é a noção que para incorporar ferramentas digitais no ensino da Matemática é necessário articular três tipos de conhecimentos: tecnológico, pedagógico e do conteúdo. Assim, aprofundando as ideias teóricas de Shulman $(1986,1987)$ sobre conhecimentos pedagógico e do conteúdo e na elaboração dessas ideias para o ensino da Matemática de Ball e seus colegas (1990, 1996), Mishra e Koehler (2006) e Koehler e Mishra (2008) avançam com um modelo 
teórico para o uso pedagógico das tecnologias digitais. Este referencial envolve uma complexa e situada forma de corpos de conhecimentos denominada: conhecimento tecnológico, pedagógico e do conteúdo (CTPC). ${ }^{5}$ Veja na Figura 1.

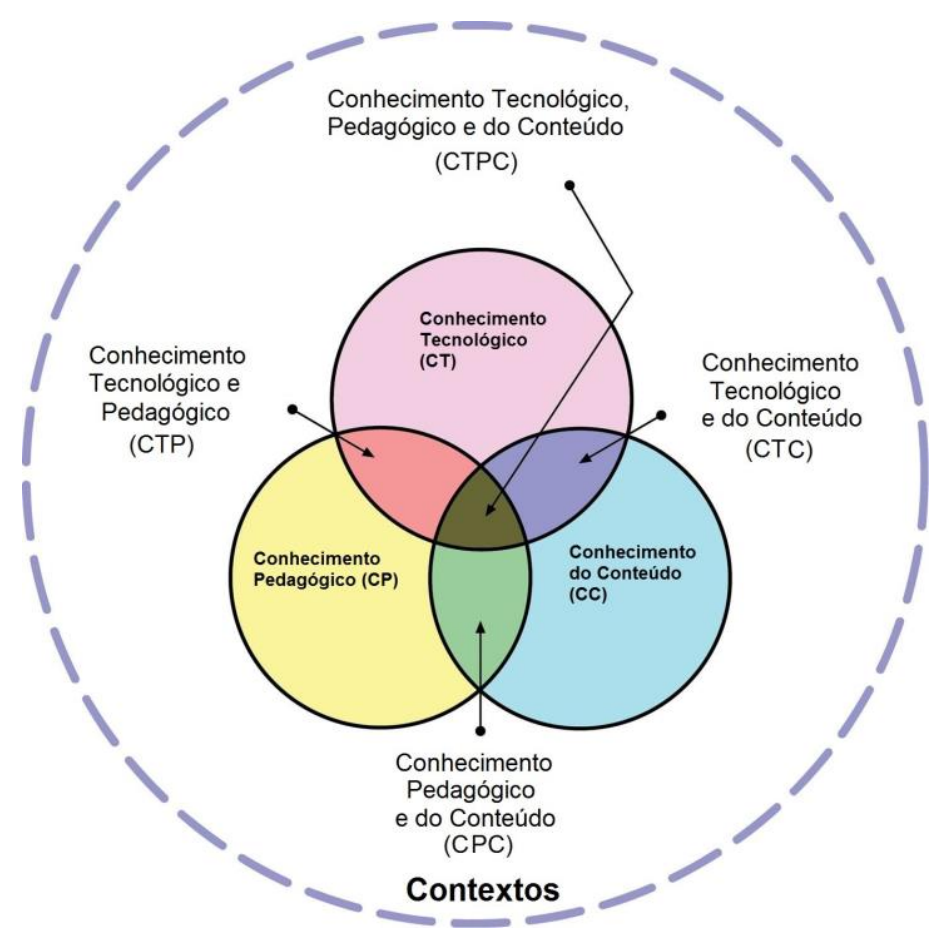

Figura 1: Um diagrama ${ }^{6}$ que ilustra o quadro conceitual de CTPC com circunferências para os três corpos de conhecimento - tecnológico, pedagógico e do conteúdo - e as quatro regiões de interseção possíveis das circunferências como as combinações possíveis dos três corpos de conhecimento.

Para Koehler e Mirsha (2008), teoricamente, em vez de ser uma coisa dada e determinada que uma pessoa descobre e contempla, o conhecimento é visto como proposições e habilidades que uma pessoa constrói e exerce. Na Figura 1, cada círculo representa um corpo de conhecimento. O círculo azul é o corpo de conhecimento do conteúdo. Ter conhecimento do conteúdo é saber o assunto a ser aprendido ou ensinado e isso é essencial para uma professora ministrar uma disciplina. Dentro do mesmo conhecimento que a professora detem, segundo Shulman (1986), devem estar incluídos os conceitos, teorias, ideias, estruturas organizacionais, o conhecimento de evidências e provas, bem como as práticas estabelecidas e abordagens para o desenvolvimento de tal conhecimento.

Além de saber a disciplina, uma professora precisa ter conhecimento pedagógico. $\mathrm{Na}$ Figura 1, isso é representado pelo círculo amarelo. Esse corpo de conhecimentos inclui saber os processos, as práticas, os métodos de ensino e aprendizagem, os propósitos gerais de educação, valores e objetivos. Também, a professora compreende como os alunos constroem conhecimentos, adquirem habilidades mentais e desenvolvem disposições positivas para a aprendizagem. A professora compreende as capacidades

\footnotetext{
${ }^{5}$ Para nós é importante sublinhar que cada uma das diferentes formas de conhecimento exista num continuum.

${ }^{6} \mathrm{O}$ diagrama está reproduzido com permissão da editora, (C) 2012 por tpack.org, e a tradução é do autor.
} 
cognitivas, sociais e teorias de desenvolvimento de aprendizagem e como estas se aplicam aos alunos em sala de aula. Além disso, a professora entende as habilidades gerais de gerenciamento de sala de aula, planejamento de aula e avaliação do aluno.

Desde o final do século passado, uma professora também pode apreciar o uso de tecnologias digitais para a construção de novos conhecimentos na sua área acadêmica. Isso nos leva ao corpo de conhecimentos, representado na Figura 1, pelo círculo cor-derosa. Segundo Koehler e Mishra (2008), uma professora que tem conhecimento tecnológico entende que tecnologias específicas têm as suas possibilidades e suas restrições. A professora aplica a tecnologia produtivamente para o trabalho e o ensino, reconhece quando esta auxilia ou dificulta a realização de um objetivo e se adapta continuamente às mudanças das tecnologias de informação e comunicação. Assim, o conhecimento de novas tecnologias evolui ao longo da vida, por meio da interação com estas tecnologias.

Finalmente, uma professora atuando com seus corpos de conhecimentos, CTPC, reconhece que não há uma única maneira para engajar seus alunos e que cada uso da tecnologia no ensino precisa ser pensado e aplicado com a especificidade do contexto da disciplina e a turma de alunos na sua sala de aula (KOEHLER e MIRSHA, 2008). Por isso, na Figura 1, os corpos de conhecimentos de CTPC existem dentro de uma área da circunferência que é o contexto.

No caso da Educação Matemática, o conhecimento CTPC existe na interseção entre conhecimentos tecnológico, pedagógico e do conteúdo matemático. Embora que entre os três corpos de conhecimentos realmente exista um equilíbrio dinâmico, há uma tensão essencial, não-separável (MISHRA e KOEHLER, 2006), mas para análise é conveniente considerá-los separadamente ou em pares. Então, dentro desta perspectiva conceitual, como se pode ver na Figura 1, podemos também focar nos três componentes: conhecimento tecnológico e pedagógico (CTP), conhecimento tecnológico e do conteúdo matemático (CTC) e conhecimento pedagógico e do conteúdo matemático (CPC).

No conhecimento tecnológico, pedagógico e do conteúdo matemático, consideramos o que é pensar matematicamente para compreender as ações que queremos observar e promover na aprendizagem. Gattegno ${ }^{7}$ (1987) propõe uma teoria psicológica sobre o que é fazer Matemática ${ }^{8}$ baseada em processos mentais. Esta abordagem apresenta dois componentes: o diálogo e a percepção.

Ninguém duvida que a Matemática se sustenta por si mesma e é o mais claro dos diálogos da mente consigo mesma. A Matemática é criada por matemáticos conversando primeiro consigo mesmo e entre si. [...] Baseado na percepção de que relações podem ser percebidas facilmente como objetos, a dinâmica que liga os diferentes tipos de relações foi extraída da mente de matemáticos e considerada por si mesma. (GATTEGNO, 1987, p. 13-14, tradução do autor)

O diálogo é algo que um indivíduo faz consigo mesmo e com os outros. Tem conteúdo composto de percepções de três possíveis elementos específicos: objetos, relações entre os objetos e dinâmicas que ligam as relações. Neste ponto, Sfard (2000, p. 47 , tradução do autor, grifo no original) teoriza que "discurso matemático e seus objetos

\footnotetext{
${ }^{7}$ Encontra-se em Powell (2007) informações biográficas sobre Gattegno.

${ }^{8}$ A expressão "fazer Matemática", utilizada originalmente por mim como to do mathematics, significa se engajar nos mesmos processos cognitivos empregados pelos matemáticos quando resolvem problemas.
} 
são mutuamente constitutivos". Podemos entender que essa dupla ação reflexivamutuamente constitutivos - inclui as relações entre os objetos e as dinâmicas que ligam essas relações. A mente humana tem a facilidade natural para perceber ou tomar consciência de objetos e relações que encontra e também de relações que ligam relações. O estudo desse jogo de relações, para Gattegno (1987), é o que distingue o trabalho de matemáticos.

Para que o diálogo sobre esses três elementos faça sentido à aprendizagem é importante que este não seja efêmero, ou seja, que tenha uma permanência com algum tipo de registro gráfico e, também, que o diálogo seja uma ação responsável e comprometida. É também essencial que seja uma atividade espontânea, mas deliberada e, pelos envolvidos, sujeita a ser repensada, relida e revisada. Esses atributos do diálogo são necessários para que este seja uma fonte para a aprendizagem e a reflexão profunda.

Essa maneira de ver a Matemática, por meio da teoria de Gattegno, nos ajuda a entender quais as ações dos aprendizes correspondem ao fazer Matemática e o que temos que observar para percebê-los fazendo matemática. As observações sobre as relações são aprofundadas com o uso da escrita, um veículo que pode resultar de uma produção colaborativa num cenário presencial ou virtual e tem implicações no aprendizado matemático e no processo de ensino e de aprendizagem em geral (POWELL e BAIRRAL, 2006).

Apresentamos as concepções e as ideias teóricas sobre a aprendizagem e o ensino da Matemática (GATTEGNO, 1987) e as relações entre os corpos de conhecimento CTPC (MISHRA e KOEHLER, 2006; KOEHLER e MISHRA, 2008) as quais farão parte da análise dos dados apresentados.

\section{O ambiente online VMTcG}

Apresentamos um ambiente virtual, com dois episódios, de professores envolvidos em duas atividades matemáticas. Destacamos seu engajamento e sua interação com os objetos, as relações entre eles e as dinâmicas ligando as diferentes relações que encontram. As atividades foram desenvolvidas online, com pequenas equipes de professores que interagiram através de um ambiente colaborativo, conhecido como Virtual Math Teams com GeoGebra-VMT ou VMTcG-(Equipes Matemáticas Virtuais com o GeoGebra, VMTcG), que incorpora uma versão do GeoGebra multiusuário e síncrona. O uso do VMTcG tem como o foco o avanço das práticas matemáticas e discursivas através da resolução, de forma colaborativa, de problemas matemáticos. De acordo com a visão de D'Ambrosio (2009, p. 10), os alunos interagem no ambiente e negociam "[...] os significados conceituais com seus colegas, buscando criar novas estratégias de soluções". Em particular, eles investigam objetos e relações matemáticas, por meio de observações, e se perguntam sobre as relações invariantes, a fim de levantar e justificar conjecturas sobre elas.

Este ambiente virtual, desenvolvido por Stahl e seus colaboradores (STAHL, 2009), o $\mathrm{VMTcG}^{9}$ tem acesso livre ao público e é fruto de um projeto financiado pela National Science Foundation nos EUA. Quando acessamos uma sala de chat encontramos um ciberespaço como está apresentado na Figura 2.

\footnotetext{
${ }^{9}$ Disponível em <www.mathforum.org/VMTLobby>.
} 
No lado esquerdo, há uma aba que dá acesso a uma janela do GeoGebra e outra aba para acessar uma lousa branca onde se pode digitar uma caixa de texto, desenhar, ou colar conteúdo de um outro aplicativo. No caso da Figura 2, abaixo das abas temos as várias ferramentas do aplicativo GeoGebra. No lado direito da janela do VMTcG, podemos ver as mensagens do chat. Acima, há um retângulo com o nome das pessoas na sala; e, no outro retângulo, abaixo da janela de chat, compõe-se a mensagem de chat antes de enviá-la.

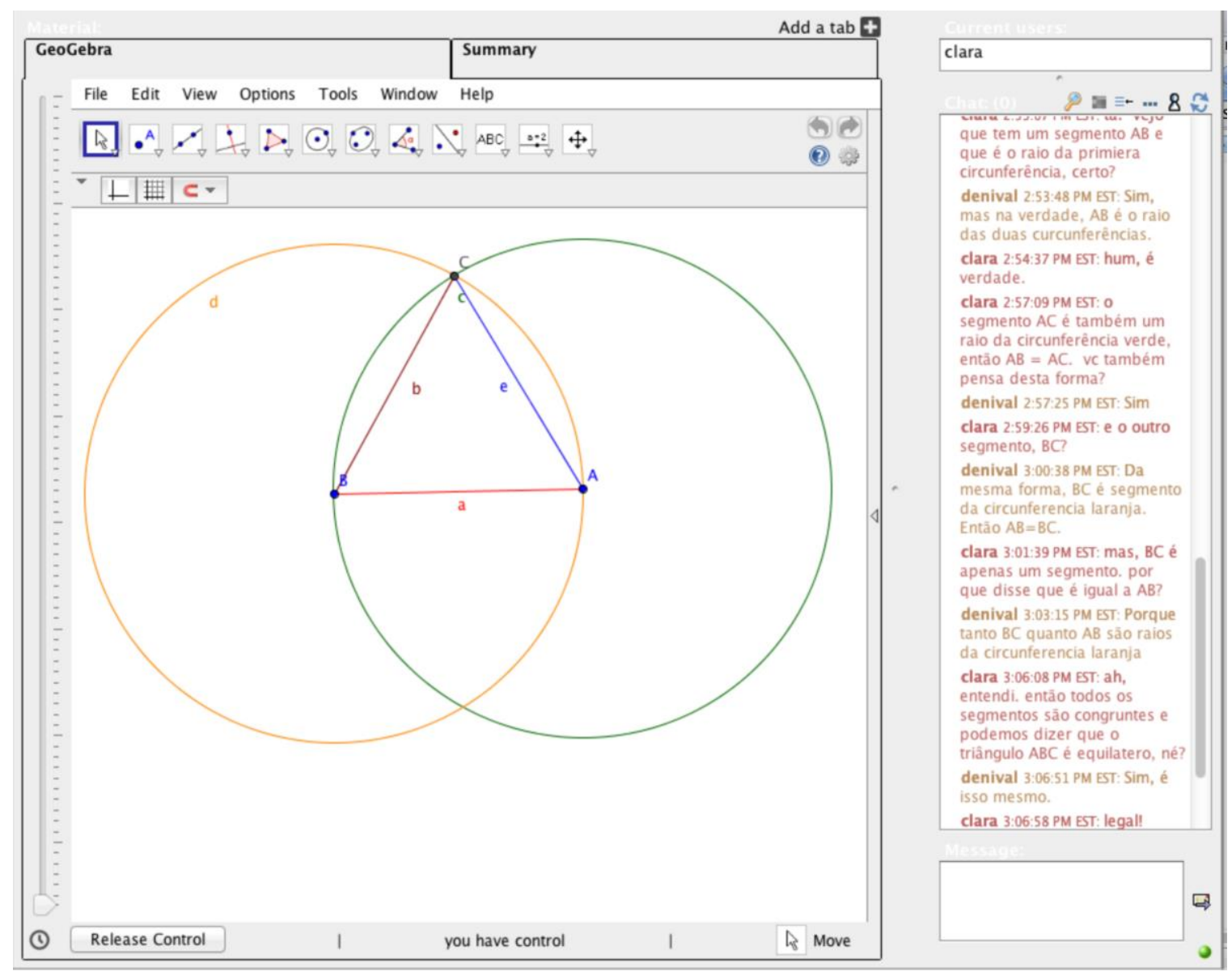

Figura 2: O ambiente VMTcG e a construção de um triângulo equilátero

\section{O projeto e Mat}

Nos Estudos Unidos, o ambiente virtual-VMTcG-está sendo usado em um projeto colaborativo, financiado pela National Science Foundation, com o nome "Computer-Supported Math Discourse among Teachers and Students" (ou "Discursos Matemáticos entre Alunos e Professores com Suporte Computacional"). O projeto colaborativo envolve pesquisadores da Drexel University, dirigido por Gerry Stahl e Steve Weimar e, da Rutgers University, dirigido pelo autor desse texto. Nós, da Rutgers University, para abreviar, chamamos o projeto como $e$ Math (ou $e$ Mat).

Um ramo do projeto $e$ Mat trata da educação continuada de professores de Matemática do Ensino Fundamental II e Médio. A disciplina é totalmente online. O 
conteúdo da disciplina tem com base a teoria CTPC de Mishra e Koehler (2006) e pedagogicamente é influenciado pelas ideias psicológicas do Gattegno (1987) sobre o que é fazer Matemática. O objetivo de evoluir os seus corpos de conhecimentos tecnológico, pedagógico e do conteúdo (CTPC). Para atingir este objetivo, trabalhamos com os professores em dois momentos distintos, cada um com duração de15 semanas. No primeiro sincronamente com VMTcG e assincronamente com Blackboard ${ }^{10}$, trabalhamos com os participantes, neste caso, como aprendizes. Depois, no segundo momento, usando as mesmas tecnologias acompanhamos e damos suporte ao trabalho que eles desenvolvem com seus alunos. O trabalho que eles fazem nas suas salas de aulas é presencial e com o VMTcG. Neste artigo, apresentamos experiências do primeiro momento.

No primeiro momento, os professores cursam uma disciplina online na qual, como aprendizes, trabalham em pequenas equipes de quatro pessoas no VMTcG, colaborando em atividades para resolver problemas matemáticos e ler artigos sobre questões pedagógicas e tecnológicas e, assincronamente e sincronamente, discutir as mesmas. Também, colaborativamente, eles desenvolvem projetos para implementar o uso do VMTcG nas suas aulas no semestre subsequente, incluindo as atividades que eles usarão com seus alunos. Em 2012, 32 professores participaram e construíram atividades em Álgebra, Geometria e Geometria Analítica. Neste ano, 14 professores estão participando e, diferentemente do ano passado, fazendo atividades focadas apenas na Geometria Euclidiana.

Neste ano, os professores são de três estados nos EUA: Nova Jersey, Pensilvânia e Ohio. A maioria deles atua em escolas urbanas com alunos de famílias economicamente carentes. Poucos tiveram experiência prévia com aplicativos de Matemática Dinâmica, em particular, com o SketchPad, Cabri, ou mesmo GeoGebra, e, além disso, não possuem um conhecimento profundo da geometria plana e isso tem implicações em como trabalham a disciplina. Jones (2000) aponta que

evidências da literatura de pesquisa sugere que muitos professores iniciantes de matemática, e, possivelmente, uma série de professores experientes, não possuem os tipos de repertórios de conhecimento do conteúdo e o conhecimento pedagógico do conteúdo de Geometria que lhes permita ensinar temas geométricos bem como poderiam. Isso indica que a atenção pode ser utilmente voltada tanto para a formação inicial de professores de matemática e seu desenvolvimento profissional contínuo. (p. 111, tradução do autor)

Nos EUA a falta de repertórios de conhecimento do conteúdo e de conhecimento pedagógico do conteúdo de Geometria tem raízes no fato de que, na maioria das vezes, os professores de Matemática do Ensino Fundamental II não estudam Geometria na sua licenciatura. Então, a última vez que estudaram Geometria Euclidiana foi no seu Ensino Médio.

Neste primeiro momento, os professores trabalham assincronamente e sincronamente. A maioria do trabalho síncrono no VMTcG é direcionado à evoluir as práticas dos professores ao colaborar em pequenas equipes de três ou quatro pessoas para discutir e resolver problemas matemáticos. As atividades que os professores são convidados para engajar são, segundo a classificação de atividades de Ponte (2003),

\footnotetext{
${ }^{10}$ Blackboard < blackboard.com> é um ambiente virtual de aprendizagem que é parecido ao Moodle.
} 
explorações e investigações. Ao longo das 15 semanas, convidamos as equipes de professores a trabalharem em 18 Tópicos. ${ }^{11}$ Cada Tópico desenvolve um assunto geométrico e tem entre três e sete atividades, sendo que cada atividade está apresentada numa Aba (Tab). As atividades variam entre construções geométricas nas quais a equipe necessita colaborar e arrastar pontos para perceber relações geométricas entre objetos construídos e relações entre relações, em particular dependências. Devem, também, estabelecer justificações sobre aquilo que os membros da equipe pensam que é verdade.

A seguir, apresentamos dois episódios que provém da disciplina ministrada para professores de matemática do Ensino Fundamental II e Ensino Médio. Os episódios são do trabalho de duas equipes de professores, cada um investigando uma atividade diferente. Nosso propósito é exemplificar que o projeto $e$ Mat contribui na construção colaborativa de CTPC dos participantes, professores de Matemática.

\section{Episódio 1: Construindo circunferências e segmentos}

O primeiro episódio refere-se também à Figura 2. Um professor e uma professora trabalham em uma sala de chat no VMTcG com uma atividade sobre segmentos e circunferências. O professor constrói uma figura na janela do GeoGebra, e a professora lhe pergunta sobre o que fez: "Tudo bem? o que fez aqui?" Ele explica: "Primeiro, eu usei a ferramenta Circle with Center through a Point (Círculo dados centro e um de seus pontos) para traçar uma circunferência com centro A.... com a mesma ferramenta, tracei a circunferência com centro em $B$, passando pelo ponto $A$ ". Pela conversa entre eles, a professora percebe a seguinte relação entre objetos: "o segmento $A C$ é também um raio da circunferência verde, então $A B=A C$. vc também pensa desta forma?". Ele responde: "Da mesma forma, $B C$ é segmento da circunferência laranja. Então $A B=B C$ ". A seguir, ela faz esta conclusão: "ah, entendi. então todos os segmentos são congruentes $e$ podemos dizer que o triângulo ABC é equilátero, né?"

É importante destacar que para se usar uma Aba na construção de algo na janela do GeoGebra, deve-se que selecionar a opção Take Control, que significa Tomar Controle. Como a versão do GeoGebra que está no ambiente é multiusuário, bem como a janela de chat, todas as ações feitas na janela do GeoGebra são vistas por todos que estão na mesma sala virtual. Também, vale destacar que o ambiente VMTcG registra todas as interações de uma sessão tanto na janela de chat como a do GeoGebra e que tem um recurso para gerar uma transcrição e um outro recurso, chamado Replayer, para rever a sessão.

Neste Episódio 1, o professor solicitou o controle para fazer a construção pedida na atividade. A professora viu o que seu colega da equipe estava construindo e queria entender como foi realizada a atividade. Com base no pedido da sua colega, ele explicou como havia construído a figura. A discussão escrita que emergiu sobre objetos, relações

\footnotetext{
${ }^{11}$ Além de dois Tópicos de ambientação do sistema VMTcG e de práticas discursivas dentro de uma equipe, nosso programa de conteúdos incluem estes dezesseis Tópicos: Construção de Triângulos, Visualizando os Teoremas de Tales e de Pitágoras, Retas Especiais, Centros de Triângulos, Relações entre Centros de Triângulos, Explorando Ângulos e Triângulos, Visualizando Triângulos Congruentes, Resolução de Problemas em Geometria I, Revisão de Triângulos, Hierarquia de Triângulos, Explorando Quadriáteros, Relações entre Quadriáteros, Resolução de Problemas em Geometria II, Resolução de Problemas em Geometria III, Atividade Individual de Transição (conexões com a Álgebra), e Transformações.
} 
entre eles, e relações de relações, levou os professores a conjeturar e justificar que, dentro da figura, existe um triângulo equilátero. Em termos de CTPC, as interações discursivas desses professores evidenciam a construção de conhecimento do conteúdo (CC) e o uso dos recursos do ambiente VMTcG mostra seu conhecimento tecnológico (CT). Mas podemos, também, ver o conhecimento tecnológico e pedagógico (CTP) com o uso de cores para que os elementos dos objetos geométricos fiquem destacados $\mathrm{e} o$ conhecimento tecnológico e do conteúdo matemático (CTC) com a maneira em que eles combinam o uso de ferramentas de GeoGebra com a percepção de relações entre objetos construída na tela.

\section{Episódio 2: Construindo colaborativamente uma demonstração do Teorema de Thales}

O segundo episódio provém do trabalho de uma equipe de professores sobre o Tópico 5-Teorema de Tales. Na primeira Aba do tópico, os professores foram convidados a discutir insights sobre os Teoremas de Tales ${ }^{12}$ e Pitágoras, derivados de seu trabalho no Tópico anterior. O Tópico 4 foi um dos únicos tópicos trabalhado não colaborativamente, mas individualmente. Naquele tópico, uma das atividades apresentadas a eles continha instruções para construir um ângulo inscrito em uma semicircunferência. Eles também foram convidados a pensar em uma demonstração para o Teorema de Tales, sabendo que Tales já havia estabelecido estes dois resultados: (1) Os ângulos da base de um triângulo isósceles são iguais, e (2) Em qualquer triângulo, a soma dos ângulos $\alpha+\beta+\gamma=180^{\circ}$.

Neste exemplo, três professores compõem uma equipe. Estão na Aba 5.01 deste tópico e decidiram discutir o Teorema de Tales, que diz: o diâmetro de uma circunferência sempre subtende um ângulo reto a qualquer ponto na circunferência (veja Figura 3). -Referindo-se a este teorema, uma das atividades do tópico anterior desafia-os para "tentar construir uma demonstração deste teorema."

\footnotetext{
${ }^{12} \mathrm{O}$ teorema de Thales: Se A, B e C são pontos sobre uma circunferência em que a linha de CA é o diâmetro do círculo, então o ângulo $\mathrm{ABC}$ é um ângulo retangular.
} 


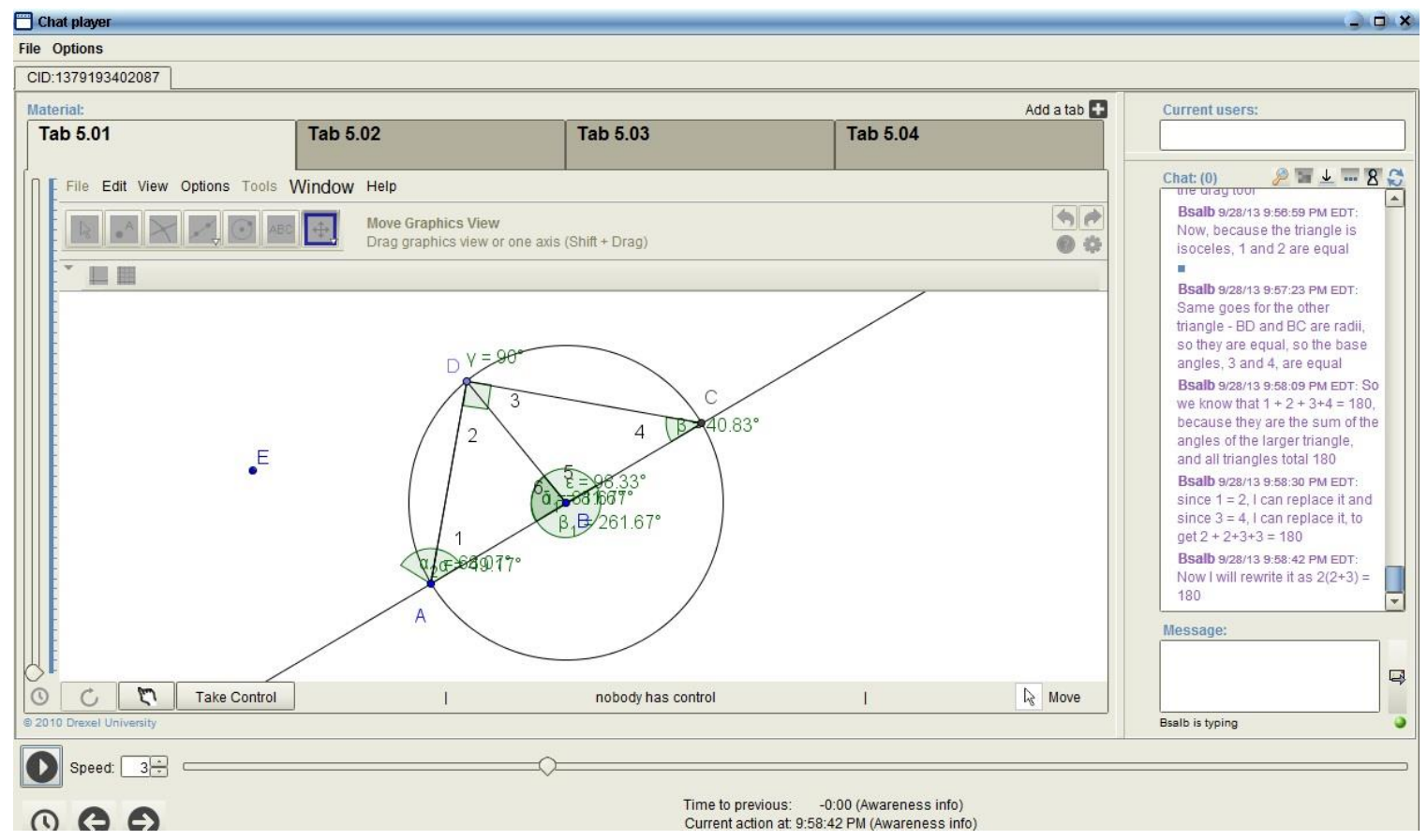

Figura 3: Um triângulo retângulo com base no diâmetro de uma circunferência.

Os três membros desta equipe são Balia, Balvir e Ira e eles decidem demonstrar o Teorema de Tales. Um membro da equipe constrói uma figura para depois falar de sua demonstração do Teorema de Tales. Enquanto ele constrói a figura, outro membro sugere objetos a serem construídos. Balvir afirma relações que ele observa entre os objetos: $\mathrm{AB}=\mathrm{BC}=\mathrm{BD}$ e a soma dos ângulos do triângulo $\mathrm{ACD}=180^{\circ}$. Ira e Balia concordam com ele. Ira assume o controle para criar medidas de ângulo e não consegue encontrar o comando para fazê-las e dá o controle para Balia, que encontra o comando. Ira solicita a Balia para indicar as medidas dos ângulos internos do triângulo ACD. Ira diz que Balia precisa clicar no sentido horário para obter as medidas dos ângulos internos. Assim que o ângulo ADC é marcado, Ira arrasta ponto D e percebe a invariância da medida do ângulo, de $90^{\circ}$. Balia afirma que a observação de Ira mostra a "realidade", mas não é suficiente como uma demonstração. Ela diz que tem uma ideia de uma demonstração com base em observações anteriores de Balvir, mas não pode continuar. Balvir retoma afirmando novas relações: como $\mathrm{AB}=\mathrm{BD}$, o triângulo $\mathrm{ABD}$ é isósceles e, como $\mathrm{BC}=\mathrm{BD}$, o triângulo $\mathrm{BCD}$ é isósceles. Ira percebe o mesmo e conclui que o ângulo $\mathrm{CBD}$ é de $90^{\circ}$. Balvir adverte Ira e diz que o ângulo CBD não é sempre $90^{\circ}$. Balia solicita e assume o controle, renomeia os ângulos com os números 1,2, 3 e 4 . Estes são os ângulos da base dos dois triângulos isósceles na Figura 3. Balia argumenta que $1+2$ $+3+4=180^{\circ}$ e que, como $1=2$ e $3=4,2+2+3+3=180^{\circ}$ ou $2(2+3)=180^{\circ}$ ou $2+3=$ $180^{\circ}$, que Ira corrige como $2+3=90^{\circ}$; ou seja, a soma das medidas dos ângulos 2 e 3 é de $90^{\circ}$. Todos parecem satisfeitos.

Neste exemplo, podemos observar vários aspectos da construção do CTPC dos professores envolvidos. O trabalho colaborativo para resolver problemas exploratórios e investigativos em que os professores evidenciam normas sociomatemáticas, é uma forma de reconhecer o conhecimento pedagógico e do conteúdo (CPC). Quando eles fazem 
conjuntamente uma demonstração de um teorema evidenciam uma evolução do seu conhecimento tecnológico e do conteúdo (CTC). Neste cenário, o conhecimento pedagógico e do conteúdo (CPC) da equipe ficou evidente quando os participantes aceitaram que as medidas dos ângulos mostram a "realidade", porém não são suficientes para uma demonstração. Nesse momento, Balia mostra a Ira que as ações de medir e arrastar são importantes estratégias para ver uma relação invariante e, nesse sentido, são úteis para perceber uma 'realidade', mas não para demonstrar que uma conjectura é verdadeira. Medição em geometria dinâmica pode ser utilizada de uma forma que potencialize o ensino de conjecturar e demonstrar (OLIVERO e ROBUTTI, 2007). A evolução desses conhecimentos do modelo CTPC são momentos que possibilitam que os professores educadores reflitam sobre como esses podem ser incorporados na sua prática pedagógica.

\section{Discussão}

Por meio de dois episódios, destacamos como é que o conhecimento tecnológico, pedagógico e do conteúdo de professores de matemática (CTPC) evoluíam. O VMTcG, um multimodal ambiente virtual, fornece oportunidades para que pequenas equipes de professores discutam, utilizando o recurso de chat, e trabalhem colaborativamente, usando um software multiusuário de matemática dinâmica, o GeoGebra. A intenção do convite para interagir no ambiente é que os professores investiguem objetos e relações matemáticas, por meio de observações e de manipulações por meio de arrastar, e se perguntem sobre as relações invariantes, a fim de levantar e justificar conjecturas sobre as mesmas.

Os participantes usam o VMT e o GeoGebra para explorar problemas exploratórios e investigativos e vivenciar esse processo numa perspectiva colaborativa. No primeiro episódio, a discussão escrita sobre objetos e relações levou os professores a conjeturar e justificar que, dentro da figura, existe um triângulo equilátero. Discursivamente, os dois professores conseguiram demonstrar o primeiro teorema coletado e demonstrado em Os Elementos de Euclides. No segundo episódio, os docentes fazem conjuntamente uma demonstração do Teorema de Tales, que também é um dos Elementos. Quando começaram, nenhum deles conseguiu demonstrar individualmente. Por outro lado, a sua cognição na equipe levou-os a construir a demonstração discursivamente. O trabalho deles é um exemplo de group cognition (STAHL, 2006) e de socially emergent cognition (POWELL, 2007). Também, esses dados evidenciam uma norma sociomatemática (YACKEL e COBB, 1996) que os professores mostram quando um deles afirmou que as medidas mostram a "realidade", porém estas não são suficientes para uma demonstração.

Os dois episódios apresentados manifestam uma construção do CTPC por professores envolvidos no $e$ Mat. Assim como, evidenciam o conhecimento tecnológico e do conteúdo e o conhecimento pedagógico e do conteúdo de uma equipe de professores

que as atividades matemáticas do $e$ Mat provocaram. Estes são elementos de dois componentes do CTPC.

\section{Considerações finais}

Começamos nossa discussão colocando em destaque dois desafios que a 
Educação Matemática enfrenta e que motivam nossa pesquisa. O primeiro desafio trata da velocidade das mudanças tecnológicas e a necessidade de que pesquisadores e educadores investiguem como aproveitar as novas tecnologias. Um estudo longitudinal sugere que a participação em projetos dessa natureza (como o $e$ Mat) pode contribuir com o desenvolvimento profissional, que se baseia nas melhores práticas atualmente aceitas e pode influenciar a aprendizagem e a prática do professor (MOUZA, 2009). Por meio do nosso projeto, $e$ Mat, estamos dando suporte aos professores que estão dispostos a se adaptarem às novas tecnologias digitais, que podem ser úteis para que os seus alunos manipulem objetos matemáticos e percebam relações entre os objetos e relações de relações.

Associado à rapidez dos avanços das tecnologias de informação e comunicação, o segundo desafio está relacionado com a necessidade de colaboração para encontrar soluções relativas ao design de projetos e desenvolvimento de novos produtos, mídias e serviços para melhorar a vida do planeta e de seus habitantes. Argumentamos que há três aspectos relativos à produção de conhecimentos que o mundo atual privilegia: (1) a interação interpessoal e social, (2) a resolução de problemas e (3) a comunicação. Esses conhecimentos são evoluídos no âmbito do $e$ Mat usando o VMTcG, colocando os professores a interagirem, colaborando para explorar e investigar situações geométricas.

Além das sessões síncronas no VMTcG, o projeto $e$ Mat contem outras atividades tais como leituras; em outra plataforma-Blackboard, apresenta um fórum para compartilhar e discutir ideias que surgem das atividades matemáticas e leituras; estudos dos históricos das interações para compreender práticas discursivas que incluem práticas matemáticas e colaborativas. A totalidade das atividades do eMat é que não só contribuem à construção do conhecimento tecnológico e do conteúdo e do conhecimento pedagógico e do conteúdo, mas como uma forma mais ampla para a evolução do CTPC dos professores.

\section{Referências}

ALAGIE, G.; ALAGIE, M. Collaborative mathematics learning in online environments. In D. Martinovic, V. Freiman, e Z. Karadag (Eds.), Visual mathematics and cyberlearning, New York: Springer, p. 23-48, 2013.

\section{BAIRRAL, M. A. Discurso, Interação e Aprendizagem Matemática em Ambientes Virtuais a Distância. Seropédica: Edur, 2007.}

BALL, D. Teacher learning and mathematics reforms: What we think we know and what we need to learn. Phi Delta Kappan, 77 (7), 500-508, 1996.

BALL, D. L.; McDIARMID, G. W. The subject matter preparation of teachers. In: W. R. Houston (Ed.), Handbook of research on teacher education (pp. 437-449). New York: Macmillan, 1990.

CHEONG, C. M.; CHEUNG, W. S. Online discussion and critical thinking skills: A case study in a Singapore Secondary School. Australasian Journal of Educational Technology , 24 (5), 556-573, 2008. 
D’ AMBROSIO, B. "Prefácio", in: Lopes, C. E. e Nacarato, A. M. (eds.). Educação matemática, leitura e escrita. Campinas: Mercado de Letras, p.9-17, 2009.

GATTEGNO, C. The science of education: Part 1: Theoretical considerations. New York: Educational Solutions, 1987.

JACOBS, J., HIEBERT, J., GIVVIN, K., HOLLINGSWORTH, H., GARNIER, H.; WEARNE, D. Does eighth-grade mathematics teaching in the United States align with the NCTM Standards? Results from the TIMSS 1995 and 1999 video studies. Journal for Research in Mathematics Education, 36, p. 5-32, 2006.

JONES, K. Teacher knowledge and professional development in geometry. British Society for Research into Learning Mathematics, 20(3), p. 109-114, 2000.

KOEHLER, M. J.; MISHRA, P. Introducing TPCK. In AACTE Committee on Innovation and Technology (Ed.), Handbook of Technological Pedagogical Content Knowledge for Educators. New York: Routledge, 2008.

LI, Y., DONG, M.; HUANG, R. Toward a Semantic Forum for Active Collaboration Learning. Educational Technology \& Society, 12 (4), p. 71-86, 2009.

MISHRA, P.; KOEHLER, M. J. Technological pedagogical content knowledge: A framework for teacher knowledge. Teachers College Record, 108 (6), p. 1017-1054, 2006.

MOUZA, C. Does research-based professional development make a difference? A longitudinal investigation of teacher learning in technology integration. Teachers College Record, 111(5), p.1195-1241, 2009.

OLIVERO, F.; ROBUTTI, O. Measuring in dynamic geometry environments as a tool for conjecturing and proving. International journal of Computers for Mathematical Learning, 12, p.135-156, 2007.

PONTE, J. P. Investigar, ensinar e aprender. Actas do ProfMat (CD, p.25-39). Lisboa APM, 2003.

POWELL, A. B. Socially emergent cognition: Particular outcome of student-to-student discursive interaction during mathematical problem solving. Horizontes, 24 (1), 33-42, 2006.

POWELL, A. B. "Caleb Gattegno (1911-1988): a famous mathematics educator from Africa?". Revista Brasileira de História da Matemática, Especial nº 1, p. 199-209, 2007.

POWELL, A. B.; BAIRRAL, M. A. A escrita e o pensamento matemático: interações e potencialidades. Campinas: Papirus, 2006. 
POWELL, A. B.; GRISI-DICKER, L. Toward collaborative, discourse-focused learning with dynamic geometry environments. Paper presented at the 12th International Congress on Mathematical Education, Seoul, Korea, 2012.

SERRADÓ, A. E-forum, a strategy for developing key competences of communication in, with and about mathematics. In GÓMEZ CHOVA, L., BELENGUER, D. M. \& TORRES, I. C. (Ed.), Proceedings of the International Conference and New Learning Technologies (EDULEARN) (p. 1-12). Barcelona: International Association for Technology, Education and Development (IATED), 2009.

SERRADÓ, A. How to question in an on-line forum to promote a democratic mathematical knowledge construction? International Journal for Mathematics in Education (Special Issue), 369-374, 2012.

SFARD, A. "Symbolizing mathematical reality into being - Or how mathematical discourse and mathematical objects create each other", In: COBB, P. et al. (eds.). Symbolizing and communicating in mathematics classrooms: Perspectives on discourse, tools, and instructional design. Mahwah, NJ: Lawrence Erlbaum Associates, p.37-98, 2000.

SHULMAN, L. S. Those who understand: Knowledge growth in teaching. Educational Researcher, 15 (2), p. 4-14, 1986.

SHULMAN, L. S. Knowledge and teaching: Foundations of the new reform. Harvard Educational Review, 57(1), p. 1-22, 1987.

STAHL, G. Group cognition: Computer support for building collaborative knowledge. Cambridge: MIT, 2006.

STAHL, G., KOSCHMANN, T.; SUTHERS, D. Aprendizagem colaborativa com suporte computacional: Uma perspectiva histórica. Boletim Gepem(53), p.11-42, 2008.

STAHL, G. (Ed.). Studying Virtual Math Teams. New York: Springer, 2009.

STIGLER, J.; HIEBERT, J. The teaching gap: Best ideas from the world's teachers for improving education in the classroom. New York: The Free Press, 1999.

YACKEL, E.; COBB, P. Sociomathematical norms, argumentation, and autonomy in mathematics. Journal for Research in Mathematics Education, 27(4), p. 458-477, 1996. 